\title{
Performance Analysis of Double Buffer Technique (DBT) Model for Mobility Support in Wireless IP Networks
}

\author{
A. A. Akintola, G. A. Aderounmu, \\ Obafemi Awolowo University, \\ Ile-Ife, Nigeria
}

\author{
M. O. Adigun \\ University of Zululand, \\ Kwadlangezwa, Republic of \\ South Africa
}

aakintola69@yahoo.com madigun@pan.uzulu.ac.za

\begin{abstract}
Existing mobility support models in cellular communications misinterpret mobility loss in cellular networks as congestion loss, thus it degrades the performance by invoking unnecessary congestion control action. In this paper, we investigated the performance of Double Buffer Technique (DBT) model for mobility support in wireless IP networks. The DBT model uses the END message and the TQRS timer to maintain the packet sequence and decrease the load on the new foreign agent when the timer expires, respectively. Also, the protocol showed improved performance degradation caused by the handover of the mobile terminal. In order to demonstrate the superiority of our scheme over the existing ones, we used the following performance metrics: packet out-of-sequence, cell loss ratio, bandwidth overhead, and suitability for real-time services. The numerical results obtained revealed that the buffer size, the waiting time, and the packet loss probabilities in the model were suitable to the wireless IP environment.
\end{abstract}

Keywords: cellular, mobility, wireless, handover, Double Buffer Technique, Internet Protocol, routing, seamless, QoS

\section{Introduction}

Future internetworks will include networks of small wireless cells populated by large numbers of portable devices. Laptop computers and cellular telephones have proven their utility, while continuing advances in miniaturization promise increasingly functional portable devices. Networks of small wireless cells offer high aggregate bandwidth, support low-powered mobile transceivers, and provide accurate location information (Caceres \& Padmanabhan, 1998). The changing needs of the business world and the availability of new technologies have led to the emergence of mobile Internet Protocol (IP) for mobility support in wireless IP networks. Mobile communications and the Internet are expected to be the main drivers for today's and tomorrow's business. This is

Material published as part of this journal, either on-line or in print, is copyrighted by Informing Science. Permission to make digital or paper copy of part or all of these works for personal or classroom use is granted without fee provided that the copies are not made or distributed for profit or commercial advantage AND that copies 1) bear this notice in full and 2) give the full citation on the first page. It is permissible to abstract these works so long as credit is given. To copy in all other cases or to republish or to post on a server or to redistribute to lists requires specific permission from the publisher at Publisher@InformingScience.org more so as new and novel kinds of value-added services over wireless broadband connections are emerging. According to Castellucia (1998) and Gustafsson, Jonsson and Perkins (2000), today's Internet does not fully support mobility. In fact, the Internet's routing and address structure prohibits 
packets addressed to a roaming mobile node from reaching it without specific support for mobility.

The increasing importance of portable computing and telecommunication applications motivate the fast development of high speed wireless networking technologies. Especially with the increasingly mainstream role of multimedia laptops, PCs, Personal Digital Assistants (PDAs) and Personal Information Assistants (PIAs) require communication techniques with higher and more flexible bandwidth (Liu, 1997). The growth of cellular radio communications in the past decade has been remarkable. Demand for cellular communications has placed heavy demand on the capacity of wireless interfaces and the network resources available. As a result, the demand for higher transmission speed and mobility is even greater.

There has been an increasing need for supporting users' mobility in today's computing environment through the Internet. One of the most important things to support terminal's mobility is the location management in the Internet. The Internet Engineering Task Force (IETF) defines Mobile Internet Protocols (IP) based on forwarding pointer concepts, which has as an extension the Route Optimization Protocol (ROP). There are some problems associated with the mobile terminal's itinery as it crosses cell boundaries especially in the midst of data transfer. One of them is the packet out-of-sequences caused by the frequent handovers of the mobile terminal. In this paper, the authors examine the mobility issues in wireless IP networks and proposed a Double Buffer Technique (DBT) model for guaranteeing the packet sequence

\section{Cellular Communication}

Cellular technology is based on geographical areas called cells. Each cell includes a base station that subscribers within the cell communicate with using two Radio Frequency (RF) links. All transmissions are full duplex, and one RF link is used for transmitting information, while the other is used for receiving (Adewale \&Falaki, 1999). Cell (i.e. Pico) are typically represented as hexagons and placed close to each other as to resemble a honey comb. The structure of a typical cellular network is as shown in Figure 1. The mobile communication device consists of hand-held phones, car phones, notebook computers, PDAs, pen-based computers, palm-top computers, and portable data collection. In this work, emphasis will be on mobile computers like notebooks since

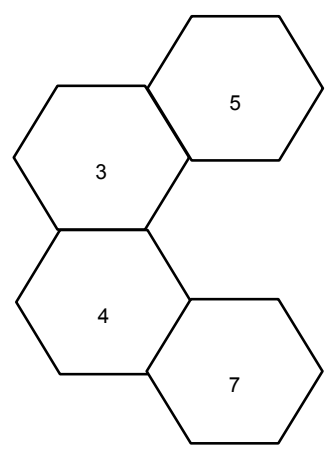

Fig. 1: A Typical Cellular Network cellular traffic in wireless IP applications is predominantly data. When these mobile units communicate with the network, they must register with the system by subscribing to a carrier service. Most carrier services have arrangement with other providers allowing users to roam. Roaming occurs when the mobile unit is outside the coverage of their cellular provider and an alternative cellular provider places the call. Wireless IP architecture offers the unrivalled advantage of providing mobile users with the connectivity they need to be productive with the flexibility of being able to change environment seamlessly.

\section{Mobility in the Internet}

To reach an individual over a network in mobile IP architectures, one has to first determine the location of the individual within the network and also the address of the individual's closest network access point. Mobility is a concept of simplifying the connection process with any desired entity. According to Lee (1998), mobility can be described as the ability to use a single logical label by any user to access an entity wherever the latter is within the network. With mobility, the 
caller does not need to determine where the entity is and the corresponding network address. In general, mobility requires an intelligent cooperation between the networks and the end points to determine the location of the destination entity.

The mobility concept can be applied to a person, a terminal or a service. Personal mobility allows connection to a person through the network independent of the location of that person. One of the basic requirements of mobile devices is that the device should be allowed to roam freely from cell to cell. This process should not require any user's intervention. In mobile IP architectures; the forwarding pointer, which is to deposit the reference of current location of mobile device to somewhere, is an efficient method for supporting mobility in the Internet.

\section{Mobile Internet Protocol (MIP)}

According to Lee (1998), the MIP is an Internet Protocol designed to support terminal mobility. Its goal is to provide the ability for a terminal to communicate with corresponding node connected to Internet regardless of their location. Mobile IP is able to track a Mobile Terminal (MT) without needing to change its long-term IP address. It has two servers i.e. the home agent and foreign agent. They are typically routers in the MT's home network, and it contains a Mobility Binding Table mapping the MT`s permanent IP address to its temporary IP address called the Care-of-Address (CoA), which stands for the current location of the MT.

Other important features of the MIP are the Agent Discovery and Registration functions. Agent Discovery is the method by which a MT determines whether it is correctly connected to its home network or to a foreign network, and by which an MT can detect when it has moved from one network to another. Registration is a process that the MT informs its CoA to the home agent, when the MT is away from home. In this work, MIP concept will be used to guarantee the packet sequence in wireless IP network environment.

\section{Route Optimization Protocol (ROP)}

In cellular networks, it is not efficient that all packets destined to a MT must be routed through the MT's home agent. This can be achieved using the triangle routing scheme. To solve this tri-

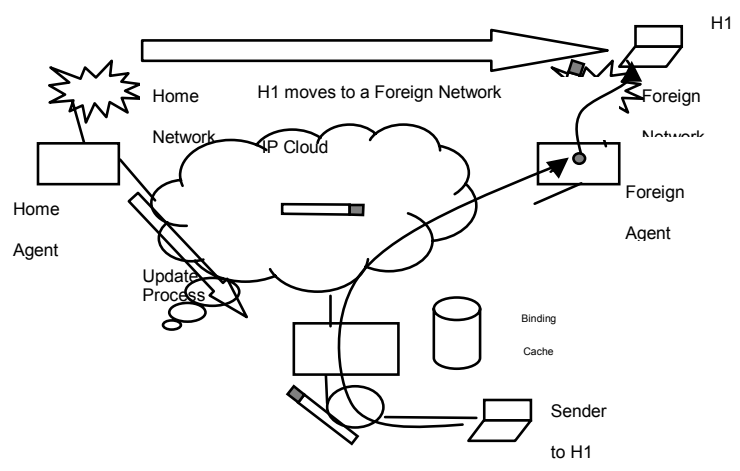

Fig. 2: Route Optimization Scheme in Mobile IP angle problem, ROP extends the concept of the basic MIP in order to achieve optimization of routing from a corresponding node to an MT through eliminating the HA. All packets destined to the MT are routed directly to the current foreign agent of the MT. Figure 2 describe the ROP scheme, in which the sender can make a "tunnel" to the foreign agent directly. The Update Process in Figure 2 represents that the home agent transfers the Registration message to the sender i.e. the corresponding node, and the foreign agent updates the Binding Cache according to the received Registration message.

\section{Agent Technology}

Agents are software programs that assist people and act on their behalf. Agents function by allowing people to delegate work to them (Danay \& Mitsuru, 1998). This is a very interesting concept that becomes even more interesting and attractive when the agents are no longer bound to the system where they begin execution. With the popularity of mobile agent technology and wireless 
cellular communications, the research into it's (i.e. agent technology) applications in wireless IPbased networks has attracted much attention recently. According to (Danay \& Mitsuru, 1998), there are several reasons for using the mobile agent concept. Some of the reasons are suitable to the implementation of mobile IP architecture, and these are:

Network load reduction

Overcome network latency

Asynchronous and autonomous execution

Dynamic adaptation

Naturally heterogeneous

Robustness

A key characteristic of the mobile agent paradigm is that any host in the network is allowed a

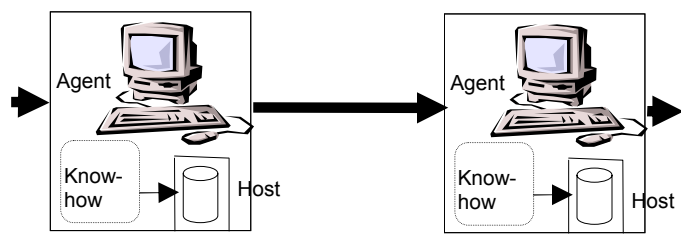

Fig. 3: Mobile Agent Paradigm high degree of flexibility to posses any mixture of know-how, resources, and processors. As shown in Figure 3, its processing capabilities can be combined with local resources. A mission-critical application may require real-time access to remote resources such as intelligent databases and perhaps even agent-to-agent negotiation. Mobile agent can employ the intentions of their creators and act and negotiate on their behalf.

\section{Problem Associated with Mobility in Wireless IP Networks}

The IETF recommends the MIP as a standard for the Internet mobility support. MIP performs the location management function for mobile devices, and controls seamless data transmission when the mobile device moves. In a cellular network, it is not unusual for a mobile device to cross cell boundaries during a data transfer using the MIP. In this case, it is possible for some packets to be

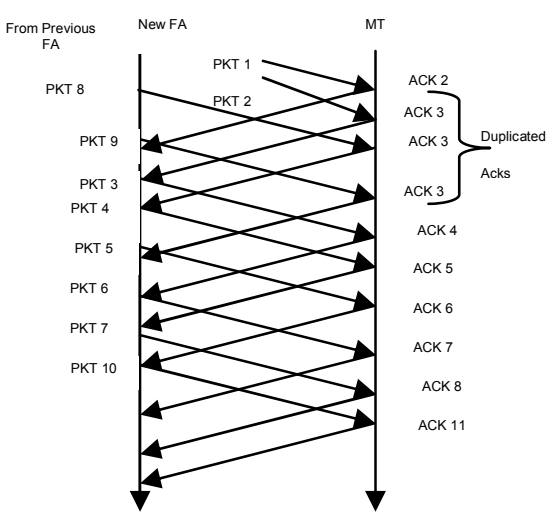

Fig. 4: Time Diagram of Packet Transmission and Acknowledgement delivered to the previous foreign agent rather than the current foreign agent due to the MT's handover procedure such as leaving a cell, joining a new cell, and processing registration. The packets that are transferred to the old foreign agent lose their path going to the MT. These packets that lose their route are called orphan packets and cause a packet loss, packet out-of-sequence, retransmissions, and duplicated acknowledgements.

Figure 4 represents the time diagram of packet transmission and acknowledgement just after terminal's handover. Since packets 8 and 9 arrive earlier than packet 3, three acknowledgements are transmitted to the sender resulting in the retransmission of packet 3.

\section{Related Researches}

In the previous section, we investigated the problems that are associated with mobility in wireless IP networks. A number of proposals have been made for the purpose of correcting the inherent 
errors. Some of these research efforts, according to Aderounmu, Akintola, Adagunodo, \& Akinde, (2004) are:

$>$ Ignoring the orphan packets- there are two methods of doing this. One is that the old foreign agent deletes the orphan packet. Another method is for the mobile agent to ignore the packet sequence. Obviously, these techniques cause packet out-of-software, packet retransmission and duplicated acknowledgements that degrade the network performance.

Waiting for the orphan packets during a fixed time - another possible solution is for the MT to wait for the orphan packets during a fixed time. After the fixed time, the MT performs packet re-ordering procedure. This can decrease the unnecessary acknowledgement and packet retransmissions. However, it is difficult to determine how long the MT waits for packets. Also, this additional mechanism becomes an overhead.

$>$ Using a "Refuge Proxy" - a refuge proxy is a stand-by agent shared by a number of mobile agents or base stations. Stranded packets, or refugees, are sent to the refuge proxy with whom the mobile agent is associated. Mobile agents conveniently fetch the astray packets from a close-by refuge proxy in the correct ordering after handover. However, as the distance between a refuge proxy and a mobile agent increases, the delay will also increase even if the old and new mobile agents are located near.

\section{System Model}

In this section, the authors discuss the system model and the functional requirements of the proposed model i.e. the Double Buffer Technique (DBT). We also looked at the model parameters and their relationship.

According to Aderounmu et al. (2004), the DBT model uses two messages i.e. the END message

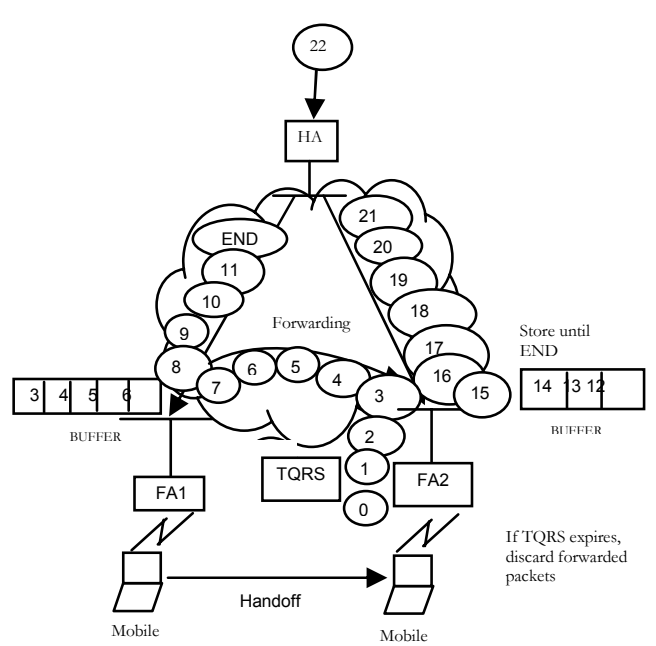

Fig. 5: The Proposed DBT Protocol / Model and the Time Quantum for Real-time Services (TQRS). Figure 5 shows the DBT protocol. The flows of signal in the DBT protocol are as shown in Figure 6. When the MT moves to the new foreign agent's network, it sends a registration message with a TQRS value to the new foreign agent. Then the new foreign agent sends the registration message to the home agent and binding update message with TQRS to the previous foreign agent. After registration in the new foreign agent's network and on the receipt of the END message, it starts transferring the stored message (i.e. packets) to the MT. Each agent has a Mobility Binding Table where it keeps the addresses of all the MTs in its network. This table resides in the Address Resolution Protocol (ARP) cache. Figure 7 shows the structure of the table. 


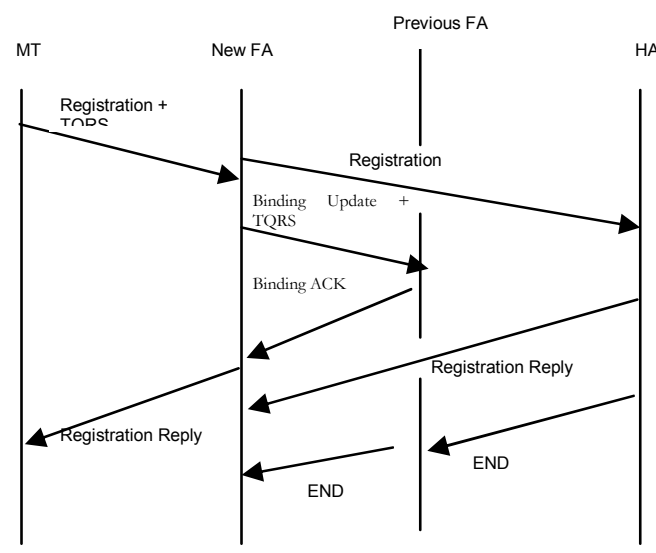

Fig. 6: DBT Signaling and Messaging Flow for MT's Handoff

\begin{tabular}{|c|c|}
\hline \multicolumn{2}{|c|}{ BINDING TABLE } \\
\hline Permanent IP address & Temporary IP address \\
\hline $\mathrm{MT}=\mathrm{A}$ & $\mathrm{CoA}=\mathrm{B}$ \\
\hline
\end{tabular}

Fig. 7: Structure of the Mobility Binding Table

\section{Model Analysis}

Let the packet arrival has a mean (i.e. average rate of packet arrival):

Mean Packet Arrival $=\lambda_{\mathrm{p}}$ packets/second

Therefore, the mean packet service rate can be represented as:

Mean Packet Service Rate, $\mathbf{P}=\mathbf{C} / \boldsymbol{\mu p}$,

where $\mathrm{C}=$ Capacity of the wireless link

$\mu \mathrm{p}=$ Number of served packet.

The inter-arrival time of activation of mobile terminals for transferring the data and the one of handover terminal has an exponential distribution with parameters $\lambda_{M}^{-1}$ and $\lambda_{H}^{-1}$ respectively. The holding times of those two kinds of cells have the exponential distribution with the mean of $\mu_{M}^{-1}$.

Let $\mathrm{K}(\mathrm{t})$ be the number of packets in the buffer and $\mathrm{A}(\mathrm{t})$ be the arrival rate of the packets. Since the packet arrival rate depends on the number of calls, the packet arrival rate $A(t)$ at the new foreign agent can be calculated by:

$A(t)=n(t) * \lambda p$

where $\mathrm{n}(\mathrm{t})=$ number of calls at a given time.

It is clear that the number of call increases when the mobile terminal is active in the region controlled by the new foreign agent or when the handover mobile terminal arrives.

It is to be noted that $\mathrm{n}(\mathrm{t})$ has a Poison Process with parameter $\lambda_{\mathrm{H}}+\lambda_{\mathrm{M}}$. Then, the system can be modeled as a two-dimensional process, characterized by $(n(t), K(t)$, where $n(t)$ and $K(t)$ is the number of call and the number of packets in the system, respectively.

The state space is represented by the set:

$$
\left[\mathrm{S}(\mathrm{n}, \mathrm{K}) \mid 0 \leq \mathrm{n} \leq \mathrm{n}_{\max }, 0 \leq \mathrm{K} \leq \mathrm{K}_{\max }\right]
$$

where $\mathrm{n}_{\max }$ is the maximum call number, and $\mathrm{K}_{\max }$ is the maximum buffer size. 
To analyze the state of the buffer, we consider the time interval starting at $t$ and ending at $t+\delta \mathrm{t}$., where $\delta \mathrm{t}$ is an infinitesimal time increment as $(\delta \mathrm{t} \rightarrow 0)$. The following possible transmissions could occur during the time interval $(\mathrm{t}, \mathrm{t}+\delta \mathrm{t})$ :

(i) a packet enters state $\mathrm{n}$ from state $\mathrm{n}-1$ with flow rate $\lambda \mathrm{P}_{\mathrm{n}-1}$;

(ii) a packet enter state $\mathrm{n}$ and from state $\mathrm{n}+1$ with a flow rate $\mu \mathrm{P}_{\mathrm{n}+1}$;

(iii) a packet leaves state $\mathrm{n}$ and enters state $\mathrm{n}-1$ with flow rate $\lambda \mathrm{P}_{\mathrm{n}}$;

(iv) a packet leaves state $\mathrm{n}$ and enters state $\mathrm{n}+1$ with a flow rate $\mu \mathrm{P}_{\mathrm{n}}$.

Flow into state $\mathrm{n}=\lambda \mathrm{Pn}-1+\mu \mathrm{Pn}+1$

Flow out of state $\mathrm{n}=\lambda \mathrm{Pn}+\mu \mathrm{Pn}$

Hence:

$$
\lambda P_{n-1}+\mu P_{n+1}=(\lambda+\mu) P_{n}
$$

But the number of

$S_{i} \rightarrow S_{i+1}=S_{i+1} \rightarrow S_{i}$

Then:

$\lambda P_{o}=\mu P_{1}$

$P_{1}=\frac{\lambda}{\mu} P_{o}$

Where $\frac{\lambda}{\mu}=$ traffic intensity

Assuming the buffer size is $\mathrm{N}$, and the sum of state probability is 1 :

$$
\begin{aligned}
& \sum_{n=0}^{N} P_{n}=1 \text { implies } P_{o} \sum_{n=0}^{N} \rho_{n} \\
& =P_{o}\left(\frac{1-\rho^{N+1}}{1-\rho}\right)
\end{aligned}
$$

Using sum of geometric progression, therefore:

$$
\begin{aligned}
P_{o} & =\frac{1-\rho}{1-\rho^{n+1}} \\
P_{n}= & p^{n}\left(\frac{1-\rho}{1-\rho^{n+1}}\right) \text { for } 0 \leq K \\
& =0 \text { otherwise. }
\end{aligned}
$$

The probability that there are $\mathrm{N}$ messages is the same as when the buffer is in state $\mathrm{P}_{\mathrm{N}}$. Therefore: 


$$
\begin{gathered}
P_{N}=P_{o} \rho^{N}=\left(\frac{1-\rho}{1-\rho^{N+1}}\right) \rho^{N} \\
=\quad \text { Probability of overflow. }
\end{gathered}
$$

Note that the packet will be lost if the packet arrives to the system whose system state is in $\mathbf{S}(\mathbf{n}, \mathbf{K})$ for $\mathbf{0} \leq \mathbf{n} \leq \mathbf{N}$. Thus, the loss probability for the packet can be written by:

$$
P_{B}=\left[\frac{\sum_{n=0}^{N_{\max }} n \lambda_{p} \pi\left(n, K_{\mathrm{Max}}\right)}{\sum_{n=0}^{N_{\max }} \sum_{k=0}^{K_{\max }} n \lambda_{p} \pi(n, K)}\right]
$$

In the DBT scheme, we will assume that $\mathrm{T}_{\mathrm{END}}$ is the END message trip time and $\mathrm{T}_{\mathrm{NEW} F \mathrm{FA}}$ be the transmission time for one packet between the home agent and the new foreign agent after handover. Each connection generates the packets with arrival rate $\lambda_{\mathrm{P}}$. The superposition arrival rate of the packet depends upon the number of connection $\mathrm{N}(\mathrm{t})$. Figure 8 shows the system model for the mobile agent supporting the DBT protocol. As shown in Figure 8 , the several packets are stored in the temporary buffer. The number of temporary buffer size is denoted as $\mathbf{i}$.

It is clear that the number of queued packet $\mathbf{i}$ is the same as the number of packets arriving during the time:

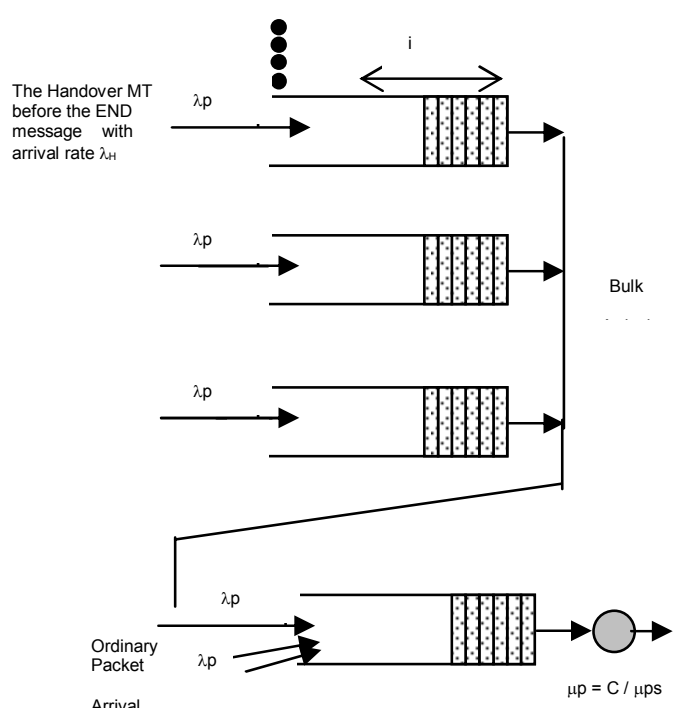

Fig. 8: System Model for Mobile Agent Supporting DBT Protocol

$\mathrm{T}_{\mathrm{Buf}}=\mathrm{T}_{\mathrm{END}}-\mathrm{T}_{\mathrm{NEW}} \mathrm{FA}$

Then, the average number of packets to be queued $\left(\mathrm{P}_{\mathrm{Q}}\right)$ can be written by:

$\mathrm{P}_{\mathrm{Q}}=\mathrm{T}_{\mathrm{Buf}} * \lambda_{\mathrm{p}}$

From Figure 8, we can easily know that the packet arrival is modeled as a bulk arrival.

\section{System Simulation and Results Analysis}

In this section; we present the system simulation, performance metrics, and the results analysis of the DBT model against one of the existing schemes i.e. Ignoring the Orphan Packets (IOP). Reason being that the architecture of the IOP model shows the best improved performance degradation for wireless IP networks. For the purpose of comparison, the following performance metrics were used: 
- Packet out-of-sequence

- Cell Loss Ratio (CLR)

- Bandwidth overhead

- Suitability for Real-time services

The simulation program was developed using the Sun Microsystems Java 2 Software Development Kit. This is because Java offers unique capabilities that are fueling the development of mobile agent system.

The simulation environment was abstracted as a two-dimensional space divided into regions managed by different agents.

The mobile terminal normally starts off from mobile agent 0 , which it registers with as its home agent.

The position of the mobile terminal is given by the vector, $\mathrm{P}$, such that:

$$
\mathrm{P}=\mathrm{x}_{\underline{1}}+\mathrm{y}_{\mathrm{i}}
$$

where $(\mathrm{x}, \mathrm{y})$ represents the $\mathrm{x}$ and $\mathrm{y}$ coordinates of the mobile terminal at any instance of time.

The motion of the mobile terminal is simulated by giving it a velocity given by:

$$
\vec{V}=\mathrm{V}_{\mathrm{xi}}+V_{y j}
$$

and the initial location of the mobile terminal is given by:

$$
\mathrm{P}_{\mathrm{o}}=\mathrm{x}_{\mathrm{oi}}+\mathrm{y}_{\mathrm{oj}}
$$

where $\left(\mathrm{x}_{\mathrm{o}}, \mathrm{y}_{\mathrm{o}}\right)$ are the initial starting location coordinates $\mathbf{x}$ and $\mathbf{y}$ of the mobile terminal. Generally, the default starting point of the mobile terminal is such that:

$$
\mathrm{x}_{\mathrm{o}}=\mathrm{y}_{\mathrm{o}}=0
$$

Figure 9 shows the possible four different positions of the mobile terminal.

In real life situation, the owner of a mobile terminal can lose contact with the cellular network if he is outside the boundaries of the cells. To prevent this in the simulation, the mobile terminal bounces off the boundaries of the simulation environment. The velocities of the mobile terminal are assigned randomly using the multiplicative congruential pseudorandom number generator.

\section{Numerical Results and Discussion}

The various performance metrics used for the comparison are discussed in the following sub-sections.

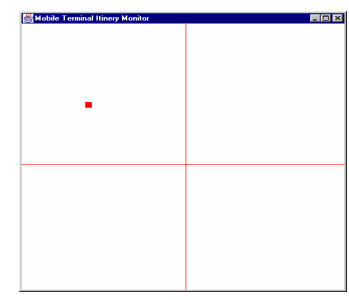

(a) MT in Home Network

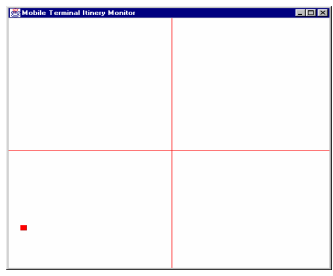

(b) MT in Foreign Network

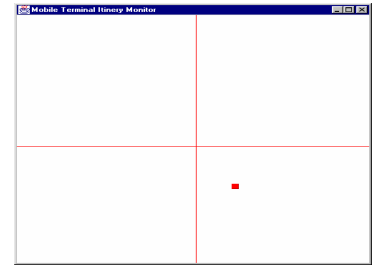

(c) MT in Foreign Network Four

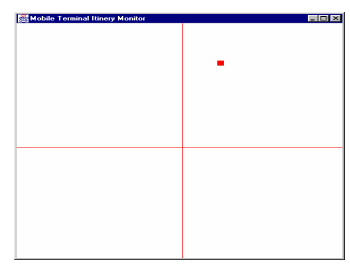

(d) MT in Foreign Network Two

Fig. 9: Different Positions of the Mobile Terminal 


\section{Packet Out-of-Sequence}

The cells reaching a mobile terminal are numbered and should be in the same order as they were generated from the cells' source. Hence, the cells generated from the cells' source can be represented by a set $\mathrm{X}$ given by:

$$
X=\left\{x: x_{i}^{\prime} \prec x_{i+1}^{\prime}\right\}
$$

where:

$\mathrm{x}$ is the set of cells generated from the source

$\mathrm{x}_{\mathrm{i}}$ is the $\mathrm{i}^{\text {th }}$ cell reaching the mobile terminal.

and

$\mathrm{X}_{\mathrm{i}}^{\prime}$ is the sequence number of the $\mathrm{i}^{\text {th }}$ cell.

$i$ is an integer value given by the set $\{0,1,2, \ldots, n-1\}$

where:

$\mathbf{n}$ is the total number of cells sent from the source.

We say that we have a cell out-of-sequence condition when the set of cells reaching the mobile terminal is given by:

$$
\begin{aligned}
& x_{\text {out }}=\left\{x: x_{i} \succ x_{i+1}^{\mid}\right\} \\
& \text {for any } i ; 0<i<n
\end{aligned}
$$

During the simulation, while varying the total number of cells used in a session, the amount of cell out-of-sequence encountered in both the DBT and the IOP schemes were measured. Figure 10 shows the amount of cell out-ofsequence for these two techniques. As could be seen, the DBT scheme never had an out-ofsequence cell condition as opposed to the IOP scheme whose cell out-of-sequence value increases as the total number of cells in a session increases. In the DBT scheme, the buffers are dynamic and could handle bursty traffic dynamically due to its scalability.

\section{The Cell Loss Ratio (CLR)}

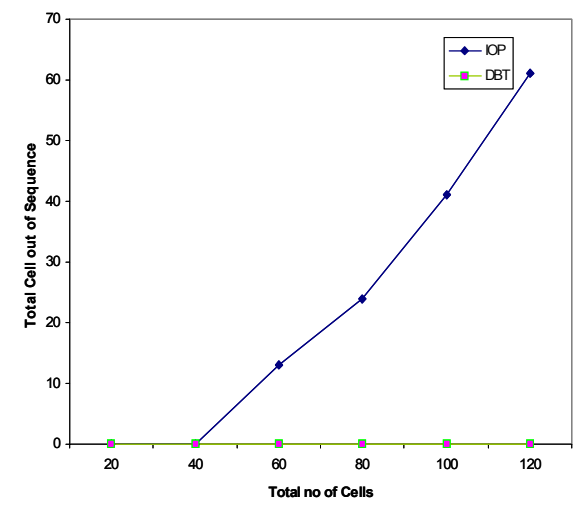

Fig. 10: Degree of Packet Out-of-Sequence in IOP and DBT Schemes

The Cell Loss Ratio (CLR) is another important measure of performance for the different cell management techniques in the wireless architecture. It is a negotiated QoS value for the various applications supported by the network protocol. The Cell Loss Ratio is given by:

$$
C L R=\frac{C_{L}}{C_{T}}
$$

where:

$\mathrm{C}_{\mathrm{L}}=$ Total number of cells lost during a transmission session

$\mathrm{C}_{\mathrm{T}}=$ Total number of cells transmitted in a session. 
The CLR is a negotiated performance parameter depending on the quality of service of the application. As can be seen in Figure 11, the dynamic and scalable buffer size in the DBT scheme does not allow cell loss during the transmission session hence; the perpetually void cell loss ratio. This is unlike the IOP scheme.

\section{Bandwidth overhead}

Each scheme has its own algorithm for cell management, which incurs a certain amount of network bandwidth overhead. Knowing that wireless connections generally have bandwidth constraints, overhead incurred by cell management schemes need to be critically analyzed in order to determine its suitability for wireless applications.

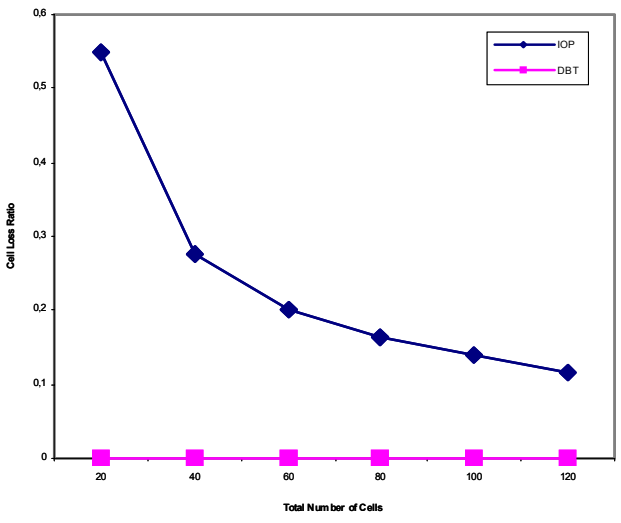

Fig. 11: Cell Loss Ratio in IOP and DBT Schemes

In the Ignore Orphan Packet (IOP) scheme, lost packets are attempted to be retransmitted on the reception of duplicated acknowledgments, this retransmission of lost cells coupled with the duplicated ACKs constitutes bandwidth overhead hence, the bandwidth overhead incurred by the IOP scheme is a direct function of the total number of repeated acknowledgements and retransmitted packets encountered in a session.

The DBT scheme uses an END message to signal the end of a segment of transmission to an agent; this protocol in the algorithm is an overhead in terms of bandwidth usage, hence it represents bandwidth overhead in the DBT scheme. The network bandwidth overhead incurred by the cells carrying the END message is lower than the overhead caused by the packet retransmissions and duplicated acknowledgements in IOP scheme. Figure 12 shows the comparison of the bandwidth overhead incurred by the DBT and IOP schemes.

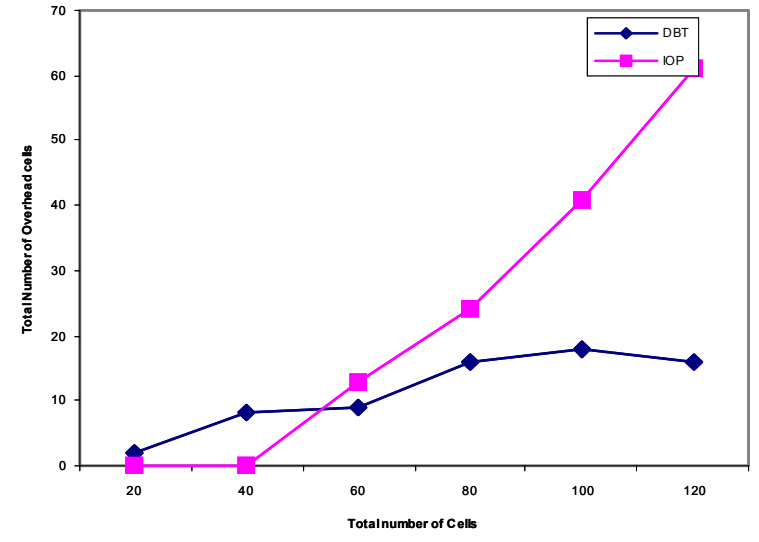

Fig. 12: Bandwidth Overhead in IOP and DBT Schemes

\section{Time Quantum for Real-time Services (TQRS)}

The TQRS was used to determine the way the orphan packets were managed. The inequalities below shows the values assigned to TQRS under various conditions.

$$
\begin{array}{lll}
0<\text { TQRS }<\text { MAX_TIMER } & \text { waiting a given time } \\
\text { TQRS }<0 & \text { no saving of out-of-order packets } & \text { ) } \\
\text { TQRS }=0 & \text { discarding the orphan packets } & \text { ) }
\end{array}
$$

From (16), it can be observed that TQRS is inversely proportional to QoS i. e. 


$$
T Q R S \alpha \frac{1}{Q o S}
$$

A constant value of unity was assumed for the purpose of the simulation. Hence;

$$
T Q R S=\frac{1}{Q o S}
$$

Also, TQRS is inversely proportional to CLR i. e.

$$
T Q R S \alpha \frac{1}{C L R}
$$

Assuming a constant of unity also, we will have:

$$
T Q R S=\frac{1}{C L R}
$$

This is because the higher the QoS, the higher the CLR due to the fact that the application will not wait for lost or orphan packets, hence; CLR is directly proportional to QoS, i. e.

\section{CLR $\alpha Q O S$ (from equations 17 and 18)}

The suitability of the DBT protocol for real-time applications was tested with various values of the TQRS. Figure 13 shows the suitability of the DBT technique for real-time services. The more real-time an application is, the lower its TQRS meaning that at the limit, an application that is a perfect real-time which cannot tolerate any delay will have a TQRS of zero. Although this is not possible in practice because that will give an infinite value to the CLR (from equation 18).

From Figure 13, the DBT scheme has an increasing cell loss ratio with decreasing TQRS as represented by the linear trend line for the cell loss ratio curve that has the equation:

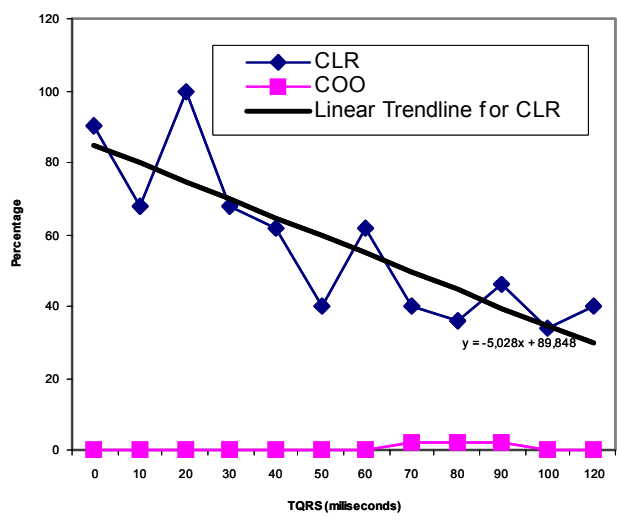

Fig. 13: Suitability of DBT Scheme for Real-time Services

$$
\mathrm{y}=-5.028 \mathrm{x}+89.848
$$

From the curve, it can be observed that it has a negative slope of -5.028. This shows that the CLR increases with decreasing TQRS (from Figure 13) making the DBT scheme less efficient for realtime services with high QoS values. Also due to the buffering, it is unsuitable for applications with low TQRS that demands less cell losses. Incidentally, the cell out-of-sequence is still managed very well even with varying TQRS. However, this is expected to work for mobile IP networks as it is not a perfect real-time technology.

\section{Conclusion and Future Research Directions}

In this paper, we have investigated the performance analysis of DBT model for mobility support in wireless IP networks. Mobile services offer advantages that can help cellular networks. The proposed DBT protocol in this research effort maintained the packet sequence using the END message and buffering in the new and old foreign networks, and it also has the TQRS timer so 
that we can decrease the load on the new foreign agent by discarding the packets when the value of the TQRS timer expires. From the numerical results obtained from the simulation, we can also deduce that the proposed DBT protocol is suitable in the practical environment.

Since mobile communications are harder to secure than traditional wire-line communications; we will, in future, investigate the automated verification of itinerant codes through program analysis to detect potential security threats.

\section{References}

Aderounmu, G. A., Akintola, A. A., Adagunodo, E. R. \& Akinde, A. D. (2004). Conceptual framework for mobile Internet protocol (IP) Agent in wireless networks. Journal of Research in Engineering, Uyo, Nigeria (Accepted).

Adewale, O.S. \& Falaki, S.O. (1999). Wireless data communication technologies in colleges and universities libraries. Proceedings of the $15^{\text {th }}$ National Conference of Computer Association of Nigeria (COAN), Vol. 10, pp. 204-211.

Caceres, R. \& Padmanabhan, V. N. (1998). Fast and scalable handoffs for wireless internetworks. Proceedings of ACM MobiCom.

Castellucia, C. (1998). A hierarchical mobile IPv6 proposal. Technical Report n 0226.

Danay, B. \& Mitsuru, o. (1998). Programming and developing Java-mobile agent with Aglet. AddisonWesley.

Gustafsson, E., Jonsson, A., \& Perkins, C. E. (2000). Mobile IP regional registration. Internet-Draft. Retrieved from http://www.ietf.org/proceedings/01aug/I-D/draft-ietf-mobileip-reg-tunnel-04.txt

Lee, D. W. (1998). A study on performance enhancement of mobile IP Agents in wireless networks. M.Sc. Thesis, Department of Information and Communication, Kwangju Institute of Science and Technology, South Korea.

Liu, C. (1997). Wireless ATM architecture design and prototyping. CIS 788 VIII Project.

\section{Biographies}

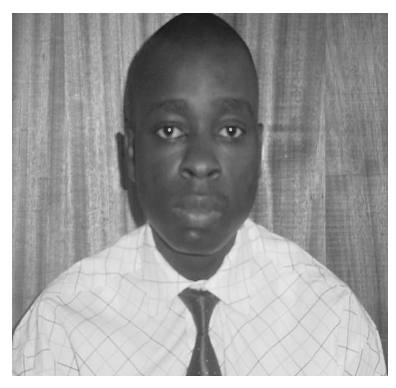

A.A. Akintola obtained his Bachelors and Masters degree in Computer Engineering and Computer Science from Obafemi Awolowo University, Ile-Ife, in 1994 and 2002, respectively. He is a registered computer engineer with Council for the Regulation of Engineering Practice in Nigeria (COREN) and also a member of Nigerian Society of Engineers (NSE). He is an author of many journal articles in Nigeria and abroad. His current research interests are in the areas of teletraffic engineering, mobile/nomadic computing, digital computer networks, hardware system studies, and computer modeling and simulation of streaming video. He is also into the areas of wireless IP networks, error control, and curriculum development. He has over 7 years of experience in teaching and research. He is currently a $\mathrm{Ph} . \mathrm{D}$. student and a lecturer at the Department of Computer Science and Engineering of the same university. 
G.A. Aderounmu holds a research degree M.Sc./PhD in Computer Science from Obafemi Awolowo University, Ile-Ife, Nigeria (1997 and 2001, respectively). He is a member of the Nige-

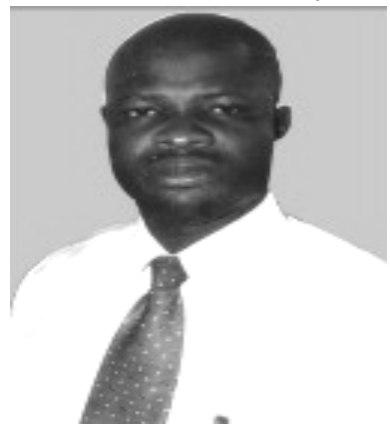
rian Society of Engineers (NSE) and is also a registered computer engineer with Council for the Regulation of Engineering Practice in Nigeria (COREN). He is also a Member of Nigerian Computer Society (NCS) and Computer Professional Registration Council of Nigeria $(\mathrm{CPN})$. He has over 12 years of experience in teaching and research. $\mathrm{He}$ is an author of many journal articles in Nigeria and abroad. His special interests include engineering education in Nigeria, curriculum development, and computer communication and network. He is a Visiting Research Fellow to the University of Zululand, Republic of South Africa. He is currently a Senior Lecturer and the Acting Head of the Department of Computer Science and Engineering of the same university.

M. O. Adigun holds a research degree $\mathrm{PhD}$ in Computer Science from Obafemi Awolowo University, Ile-Ife, Nigeria, which he obtained in 1989. Currently, he is a Professor and Head, Department of Computer Science, University of Zululand, Republic of South Africa. His research interest include Software Engineering, Mobile Computing, Modeling and Simulation, and Performance Analysis of Computer System 